\title{
Correlating mechanical properties with aggregation processes in electrochemically fabricated collagen \\ membranes
}

M. Ramesh Kumar, ${ }^{\dagger}$ Erika F. Merschrod S., ${ }^{* \dagger}$ and Kristin M. Poduska $\dagger^{*, \neq}$

Department of Chemistry, and Department of Physics and Physical Oceanography, Memorial

University of Newfoundland, St. John's, NL, AlB 3X7, Canada

E-mail: kris@mun.ca,Phone:(709)737-8890,Fax:(709)737-8739;

erika@mun.ca,Phone:(709)737-8765,Fax:(709)737-3702

\footnotetext{
${ }^{\dagger}$ Department of Chemistry

Department of Physics and Physical Oceanography
} 


\begin{abstract}
We show that mechanical stiffness is a useful metric for characterizing complex collagen assemblies, providing insight about aggregation products and pathways in collagen-based materials. This study focuses on mechanically robust collagenous membranes produced by an electrochemical synthesis process. Changing the duration of the applied electric field, or adjusting the electrolyte composition (by adding $\mathrm{Ca}^{2+}, \mathrm{K}^{+}, \mathrm{Na}^{+}$or by changing $\mathrm{pH}$ ), produces membranes with a range of Young's moduli as determined from force-displacement measurements with an atomic force microscope. The structural organization - characterized by UV-visible spectroscopy, Raman spectroscopy, optical microscopy and atomic force microscopy - correlates with the mechanical stiffness. These data provide insights into the relative importance of different aggregation pathways enabled by our multi-parameter electrochemically-induced collagen assembly process.
\end{abstract}

Keywords: Young's modulus, force curves, protein aggregation, fibrillogenesis, collagen, electrosynthesis, Raman spectroscopy 


\section{Introduction}

Knowledge of the mechanical properties of biological tissues is instrumental in understanding how structure affects their function. Tendons, for example, are aligned collagen-based connective tissue whose elastic properties have been widely studied at macroscopic length scales. ${ }^{1-3}$ However, mechanical property investigations at smaller length scales, relevant to the local environment experienced by a cell, require smaller probes. Force-displacement curves obtained with atomic force microscopy (AFM) cantilevers, whose tips have radii of curvature on the order of tens of nanometers, can be used in conjunction with appropriate mathematical models to describe the tipsample contact mechanics and to estimate the Young's modulus and other structural parameters in a range of materials. ${ }^{4}$ Here, we show that mechanical property data obtained from AFM forcedisplacement curves can be used to distinguish possible formation pathways of complex collagen assemblies.

Collagen is a structural protein whose mechanical properties are intimately related to the assembly and aggregation of its monomeric building blocks. Its hierarchical aggregation process, fibrillogenesis, is fundamental to the in vivo development of collagenous connective tissues. ${ }^{5}$ These fibrils and smaller protofibrils are structurally and mechanically distinct from the less stiff, nonspecifically aggregated collagen that can form under some conditions, as presented schematically in Figure 1. Fortuitously, fibril formation can be replicated in a laboratory environment, ${ }^{5}$ which can aid development of synthetic collagen-based scaffolds for tissue repair and regeneration applications. ${ }^{6,7}$ Strategies for developing such scaffolds require controlled collagen assembly for reliable function, including control over packing density, elastic deformability, and the final size of the construct. These factors can affect bioactivity through mechanical response, or as a result of bioavailability issues arising from the porosity of the material.

Collagen aggregation and fibrillogenesis have been directed in vitro by stimulating alignment prior to or during aggregation via fluid flow, mechanical extrusion, microfluidic channels, or anisotropic chemical nanopatterns. ${ }^{1,8,10-13}$ An alternative class of approaches for providing high orientational order and packing density involves manipulating the electrochemical environment 


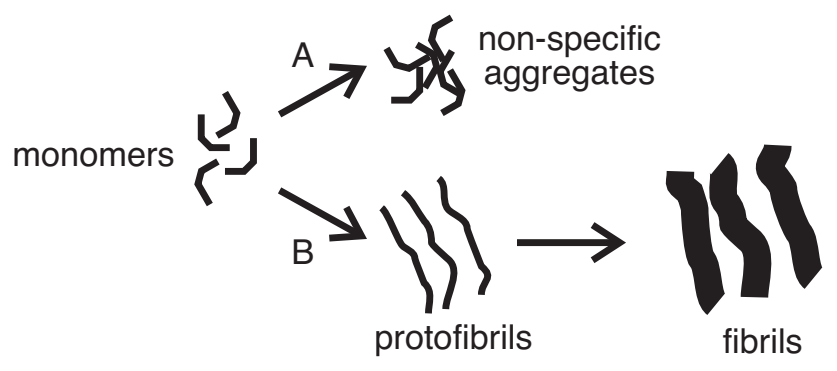

Figure 1: A schematic diagram of possible membrane formation pathways. Monomers can either aggregate (A) non-specifically or (B) hierarchically to form protofibrils and then fibrils. Nonspecific aggregates do not lead to fibril formation.

of collagen molecules. Marino and Becker ${ }^{14}$ reported that the electrolysis of a collagen solution could induce the formation of an opaque fibrous material, with aggregation induced by a local $\mathrm{pH}$ increase at the cathode. More recently, our group has reported the formation of macroscopic and microscopic $2 \mathrm{D}$ collagen membranes by a similar process, ${ }^{15}$ while othershave made collagen bundles in the presence of electric fields ${ }^{3,16}$.

In this work, we demonstrate that AFM force measurements coupled with spectroscopic data allow us to understand how electrochemical synthesis parameters influence collagen membrane formation, toward the goal of a tailored membrane structure for scaffold applications.

\section{Experimental method}

\section{Sample preparation}

Our experiments were designed to investigate the effects of time, $\mathrm{pH}$, and electrolyte composition on an electrochemically controlled collagen membrane formation process. The electrolyte contained collagen (Type I collagen monomers with a final concentration $0.07 \mathrm{mg} / \mathrm{ml}$ from $3 \mathrm{mg} / \mathrm{ml}$ acidic Vitrogen stock solution, Inamed Biomaterials), ultrapure water (18.2 $\mathrm{M} \Omega \cdot \mathrm{cm}$, Barnstead Nanopure), and sufficient $\mathrm{NaOH}$ (EMD Chemicals, ACS reagent grade) to reach $\mathrm{pH} 7$, unless otherwise noted. Electrolytes more alkaline than $\mathrm{pH} 7$ can lead to collagen denaturation, so our study focused on $\mathrm{pH}$ variations in the acidic regime. In some experiments, different concentrations 
(5-100 mM) of $\mathrm{CaCl}_{2}, \mathrm{KCl}$, and $\mathrm{NaCl}$ were added (at $\mathrm{pH}$ 7). All experiments were conducted at ambient temperature.

The electrochemically induced aggregation was carried out in a two-electrode electrochemical

cell described in more detail elsewhere. ${ }^{15}$ A potential of $8 \mathrm{~V}$ (unreferenced) was applied with a potentiostat (Pine 366A) for durations between 15 and 60 minutes. The resulting current data were recorded using a computer-based data acquisition program (National Instruments LabVIEW and NI-PCI 6014 digitizing board) with an interface designed in-house. Prior to subsequent measurements, membranes were removed from the cell, rinsed with ultrapure water, and air-dried on a glass substrate under ambient conditions. Aliquots from the electrolyte were also collected, deposited on a glass substrate, and air-dried under ambient conditions. Variations in ambient humidity and temperature did not lead to statistically significant changes in membrane stiffness.

\section{Optical characterization}

The membrane formation process was monitored in real time during some experiments with a Leica DM2500 optical microscope and polarized light. Raman scattering spectroscopy (Jobin Yvon Horiba LabRAM, confocal, $532 \mathrm{~nm}$ excitation) on dried membranes tracked changes in intramolecular and intermolecular bonding, which are indicators for degree of collagen aggregation and/or denaturation. To assess how much of the collagen from the electrolyte was incorporated into the membrane, we used UV/Vis spectroscopy (Ocean Optics, Inc. Chem2000) on the postdeposition electrolyte to measure relative changes in absorption compared to an ultrapure water reference.

\section{Atomic Force Microscopy}

An atomic force microscope (MFP-3D AFM, Asylum Research) was used for thickness measurements, topographic mapping and force-displacement measurements. Experiments were performed at ambient temperature using silicon probes (MikroMasch NSC35) with spring constant $\sim 17 \mathrm{nN} / \mathrm{nm}$, as determined by the thermal noise method. ${ }^{17}$ Membrane thicknesses were assessed 
by AFM measurements at the edges of the samples, with measurement sites selected where optical microscope indicated that the membrane was not folded over. Topographic images were acquired using tapping imaging mode at a scan rate of $0.5 \mathrm{~Hz}$. Before beginning indentation measurements on the sample, force curves were collected on a bare glass substrate to calibrate the deflection sensitivity of the instrument. Force curves were then collected at different locations on the collagen membrane within a $25 \mu \mathrm{m}^{2}$ area, starting $\sim 200 \mathrm{~nm}$ above the sample surface and indenting $\sim 100 \mathrm{~nm}$ after initial contact with the sample. Force curve shape indicated that the measurements were not affected by the underlying substrate: the curvature of the extension and retraction curves was accounted for entirely by probe shape and did not show evidence of coupling with the harder substrate. ${ }^{4}$ These raw cantilever deflection voltage $v s$. probe displacement measurements were then converted into force-separation relations using the cantilever deflection sensitivities and the cantilever spring constants. We use a simplified model for the material response as purely elastic, neglecting any viscoelastic contributions and using only the retraction portion of the forcedisplacement curves to eliminate contributions from plastic deformation. Representative force curves and additional details on their analysis are provided in the Supporting Information.

We note that the mechanical data were collected on dried membranes to avoid artifacts introduced by capillary forces. Force curves obtained on membranes that were never dried (or membranes that were rehydrated) are dominated by capillary forces, while measurements on dried membranes are free of these strong artifacts. Measurements on wet membranes conducted entirely under fluid present additional problems with inconsistent adhesion of the membrane to the underlying support, and with strong adhesion between tip and membrane. Membranes would likely be rehydrated when used in scaffolding applications, and this would change their Young's moduli. ${ }^{9}$ However, our interest here lies in using mechanical measurements to model the formation process of the membranes, and this data is obtained most consistently with dried membranes. 


\section{Modeling stiffness}

We employed two theoretical models that are widely used to extract Young's modulus. A hyperboloid tip shape ${ }^{18}$ is assumed in a Hertzian contact model, ${ }^{19}$ while the model of Oliver and Pharr ${ }^{20,21}$ utilizes the shapes of retraction curves to determine an effective indenter shapes. By treating all data with both contact models, we confirmed that observed qualitative trends for changes in stiffness are not dependent on the specific details of the probe-sample contact modeling. Additional details and representative analyses from both models are included as Supporting Information.

\section{Hertzian model}

The force-indentation relation for a hyperboloidal contact ${ }^{18}$ is given by Equation 1

$$
F=\frac{E a^{3}}{\left(1-\sigma^{2}\right) R}\left[\xi^{2}+\left(1-\xi^{2}\right) \frac{R \delta}{a^{2}}\right]
$$

where $F$ is the load force, $\xi$ is $R \cot (\alpha) / a, a$ is the contact area radius, and $\alpha$ is the tip conical angle ( $30^{\circ}$ according to manufacturer specifications for our indenters), $R$ is the probe's radius of curvature, $E$ is the Young's modulus, $\sigma$ is the sample's Poisson ratio $(0.3$ is a reasonable value for biological fibrils ${ }^{4}$ ), and $\delta$ is the indentation depth and is equal to

$$
\delta=\frac{a^{2} \xi}{2 R}\left[\frac{\pi}{2}+\arctan \left(\frac{1}{2 \xi}-\frac{\xi}{2}\right)\right]
$$

An accurate calculation of the indentation depth $\delta$ would require precise knowledge of the probesample contact point. Instead, one can use an estimated tip-sample separation ${ }^{4}(\Delta)$ offset from the true indentation depth by a constant $C: \Delta=C-\delta$. It is then possible to rework Equation 1 so that the Young's modulus can be determined independently of the exact point of probe-sample contact. 


\section{Oliver-Pharr model}

The Oliver-Pharr model ${ }^{21,22}$ adopts a slightly more complex model for the indentation process. The effective Young's modulus $E$ is related to the sample stiffness $\left(S_{\text {material }}\right)$ and contact area (A) by

$$
E=\frac{S_{\text {material }}}{2} \sqrt{\frac{\pi}{A}},
$$

when assuming an indenter that is approximately axially symmetric. ${ }^{20}$ The contact involves two springs connected in series: one for the AFM cantilever (spring constant $K_{c}$ ) and another for the sample (pseudo-spring constant $S_{\text {material }}$ ). These two spring constants are related to the slope of the force $v s$. indentation depth plot $\left(S_{\text {expt }}\right)$ obtained from force curve measurements.

$$
\frac{1}{S_{\text {material }}}=\frac{1}{S_{\text {expt }}}-\frac{1}{K_{c}}
$$

The contact area $A$ is determined by accounting for both the geometry of the tip and the plastic deformation from the extension process (which affects the tip-sample contact upon retraction). Fits of our retraction curves (described in the Supporting Information) indicate that our contact area is effectively parabolic, so we use a contact area given by

$$
A=2 \pi R h_{c}-\pi h_{c}^{2}
$$

where $R$ is the radius of curvature of the indenter and $h_{c}$ is the contact depth.

The contact depth upon retraction is affected by plastic deformation from the extension process. If the sample is permanently indented during extension, the retraction process will occur over a shorter distance and will involve a different surface geometry and hence a different tip-surface contact. In principle, it would be possible to determine the retraction distance by measuring the height difference between the point of first tip-sample contact on the fresh sample (during the tip extension procedure) and the point of last tip-sample contact on the newly deformed sample (during the tip retraction procedure). However, adhesion effects could cause the height at which 
the tip disengages from the sample to be different from the true sample height. A solution that addresses the changes in both distance and shape during retraction estimates how much sinking deformation should occur to the sample ${ }^{21}$ based on the maximum force applied during indentation $F_{\text {max }}$, the spring constant of the material being indented $S_{\text {material }}$, and the effective shape of the indenter $\varepsilon .^{22}$ The true contact depth $h_{c}$ is then the total measured height change $h$ corrected for this sinking depth.

$$
h_{c}=h-\left(\varepsilon \frac{F_{\text {max }}}{S_{\text {material }}}\right)
$$

Each retraction curve is fitted (as described in the Supporting Information) to obtain $\varepsilon$, all of which are close to the 0.75 value of a paraboloid tip. ${ }^{21}$ Since it is impractical to assess the radius of curvature for each indentation, our calculations are based on manufacturer specifications $(R=$ $20 \mathrm{~nm}$, MikroMasch, NSC 35). We note that variations in tip radius $\pm 10 \mathrm{~nm}$ lead to less than $10 \%$ change in the calculated Young's moduli using Eq. 3 and 5.

\section{Results}

\section{Membrane formation}

The speed of membrane formation can be adjusted by changing cell geometry, applied voltage or electrolyte composition. The onset of film formation, viewed easily with the naked eye, is very reproducible for experiments using the same electrode geometry and spacing (for example, $\sim 9$ minutes for $\mathrm{pH} 7$ electrolytes exposed to $8 \mathrm{~V}$ from parallel plate electrodes $2.5 \mathrm{~cm}$ apart). Larger voltages or closer electrode spacings lead to faster film formation. Electrolytes whose initial $\mathrm{pH}$ values are further from the isoelectric point of collagen $\left(\mathrm{pH} \sim 6.5\right.$ in our experiments ${ }^{15}$ ) require longer times to form the collagen membranes (Figure 2a). Finally, film formation time decreases slightly with increasing ionic strength of the electrolyte. Figure $2 b$ illustrates this effect with the addition of $\mathrm{KCl}$; similar trends were observed with $\mathrm{CaCl}_{2}$ and $\mathrm{NaCl}$.

Although electrolyte composition differences can change how rapidly the initial membrane 

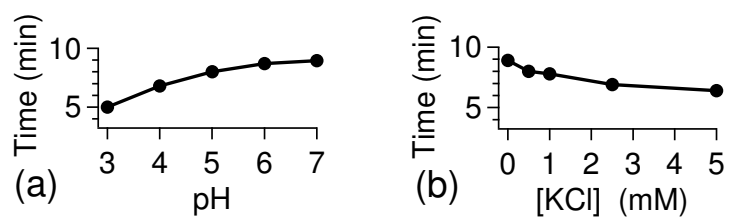

Figure 2: More acidic pH values (a) and lower ionic strengths (b) increase the amount of time required for membrane formation. The uncertainty estimates associated with each data point are contained within the size of the markers, and the lines connecting the data points serve as guides to the eye.

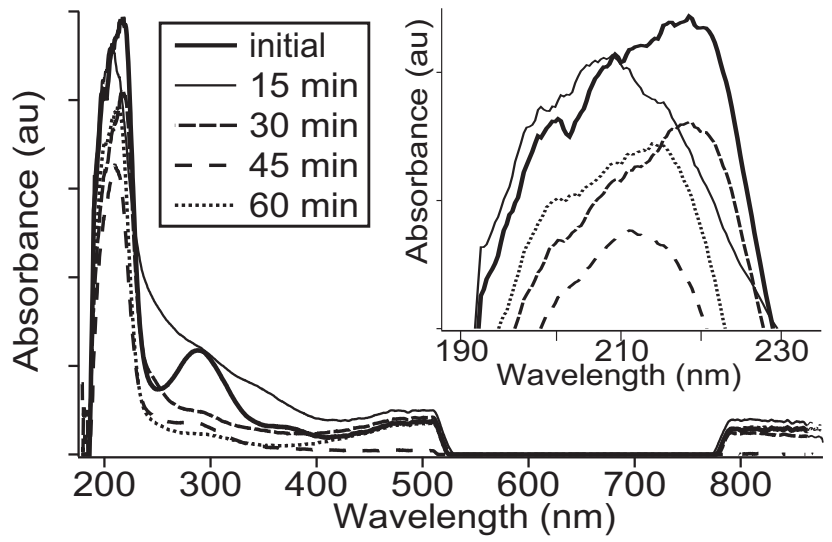

Figure 3: UV-Vis absorption spectra of post-deposition electrolytes show that longer exposure to the applied potential leads to a decrease in the collagen-related absorbance peak near $200 \mathrm{~nm}$ (inset), corresponding to more collagen incorporation into the membrane. The marker spacings on the ordinate axes are consistent for the main graph and the inset.

forms, the membrane continues to grow over time as long as the applied cell voltage remains. Figure 3 shows absorbance $v s$. wavelength data from the post-synthesis electrolytes (initial $\mathrm{pH} 7$ ) after exposure to the applied potential for different amounts of time. All initial and final electrolytes were colorless, so no peaks were obtained in the visible region. However, the intensities of the broad peaks near $200 \mathrm{~nm}$ (originating from peptide bonds) and $300 \mathrm{~nm}$ (due to aromatic amino acid side chains, prominent only in the initial electrolyte prior to aggregation) decrease with increasing electrosynthesis time, suggesting that collagen is continually removed from the electrolyte as it is incorporated into the membrane. We note that there was little difference in absorbance spectra as a function of electrolyte $\mathrm{pH}$ or ion concentration for the same times and applied potentials. Membrane thickness also increases with electrosynthesis time, consistent with the optical absorption data trends in Figure 3. AFM data indicate thicknesses ranging from $240 \pm 20 \mathrm{~nm}$ at 15 minutes to 
$420 \pm 50 \mathrm{~nm}$ at 60 minutes. We conclude that increasing the time allowed for electrosynthesis is more effective at growing the membrane than merely changing the ionic content of the electrolyte. All subsequent data presented here will correspond to films electrosynthesized for 30 minutes, unless otherwise indicated.

\section{Correlating mechanical stiffness with fibril content}

We quantified Young's moduli for electrosynthesized collagen membranes based on data from AFM indentation curves on dried membranes. The models we propose for membrane formation and stiffness control arise from assessments of relative changes in the Young's moduli, rather than on their absolute values, but it is reassuring that the values for our dried membranes do lie within the broad range $(0.2-3 \mathrm{GPa})$ of Young's moduli that have been reported for collagen fibrils. ${ }^{23-25}$
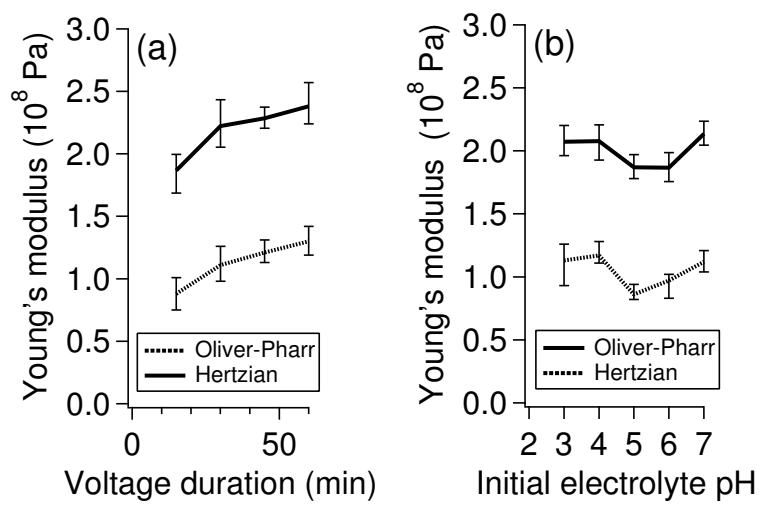

Figure 4: Young's modulus values for electrosynthesized collagen membranes change as two parameters were varied independently: (a) duration of applied voltage (at $\mathrm{pH} 7$ ) and (b) initial electrolyte $\mathrm{pH}$ value (for 30 minute voltage duration). Error bars corrspond to a set of measurements made with the same tip on the same sample. Trends are qualitatively similar using either Hertzian or Oliver-Pharr models for tip-sample-contacts.

Figure 4 shows representative data that confirm that electrosynthesis conditions can influence membrane stiffness. These data also indicate that there is a striking spatial uniformity in the mechanical response of membrane. While AFM indentation is intrinsically a local measurement, the stiffness variations across a given membrane (error bars) are smaller than the changes observed under different synthesis conditions (trend lines). To understand this, it is important to recognize 
that an indentation with our probe will access a depth 100-200 nm and initiate a rather large contact area (with diameter 100-200 nm) involving a network of monomers and/or fibrils. Therefore, although more mature fibrils could be larger than the tip diameter $(\sim 20 \mathrm{~nm})$, indentation does not necessarily measure the modulus of a single fibril. Figure 4 also demonstrates that moduli calculated from Hertzian (Equation 1) and Oliver-Pharr (Equation 3) models showed qualitatively similar trends for all samples. This suggests that the stiffness trends we observe are not dependent on the details of how the tip-sample interactions are modeled.
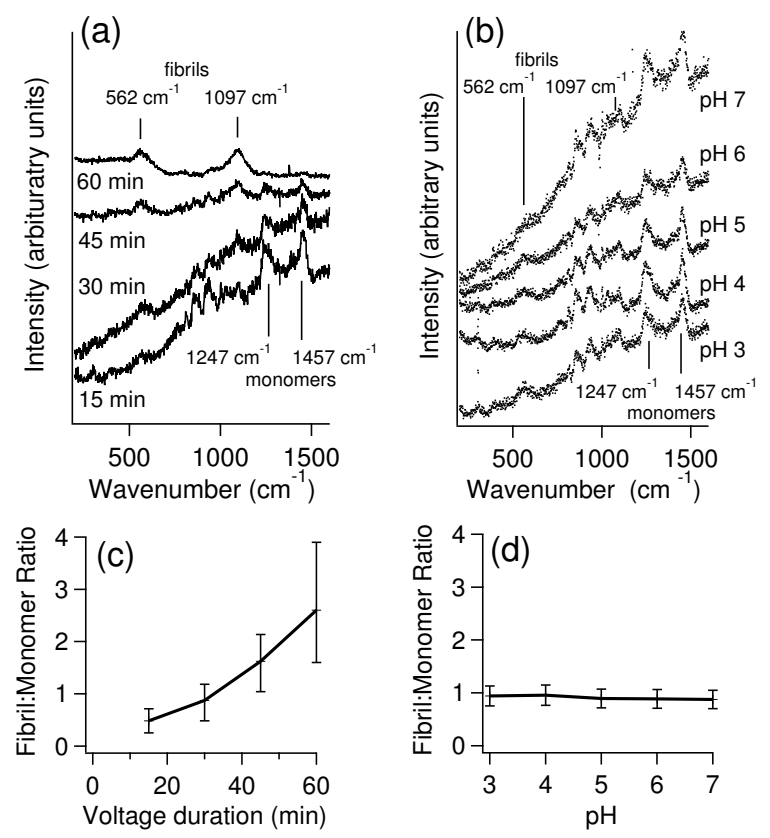

Figure 5: Raman spectra from collagen samples prepared with (a) different durations of applied voltage (all at $\mathrm{pH}$ 7) and (b) electrolytes with different initial $\mathrm{pH}$ values (all with 30 minutes of applied voltage). Spectra are offset along the intensity axis for clarity. Estimates of fibril to monomer ratio based on peak height comparisons from these spectra show (c) more distinctive fibril signatures for longer duration of applied voltage, and (d) no significant correlation between fibril content and $\mathrm{pH}$. The increasing background level for higher wavenumbers is generally characteristic of monomeric samples with some denaturation, and is therefore more prevalent for samples with higher monomer content.

The Young's modulus shows a marked increase ( $\sim 40 \%)$ over the first 30 minutes of membrane formation (Figure 4a). (We note that this trend cannot be attributed to substrate artifacts affecting probe indentation, since membranes are also increasing in thickness over time.) Instead, we attribute this stiffness increase to higher fibril content in the membrane, confirmed with Ra- 
man scattering data (Figure 5a,c) based on assignments of the amide III regions $\left(560 \mathrm{~cm}^{-1}\right.$ and $1240-1270 \mathrm{~cm}^{-1}$, associated with fibrilized collagen) and the C-N stretch regions $\left(1095 \mathrm{~cm}^{-1}\right.$ and $1454 \mathrm{~cm}^{-1}$, predominant for monomeric collagen). ${ }^{26,27}$ Because the Raman peaks of interest are broad (particularly for fibrils) and overlap with other peaks (particularly for monomers), fitting the peaks to calculate areas would introduce more parametrization than the data could justify. Peak height was therefore a more consistent and reliable means to approximate the relative changes in monomer and fibril content.

Another factor that has a more moderate effect on membrane stiffness is the initial $\mathrm{pH}$ of the starting electrolyte. Adjusting the initial electrolyte $\mathrm{pH}$ to 5-6 yields a slight decrease in the membrane Young's modulus (Figure 4b). This trend is harder to correlate with the membranes' relative monomer and fibril contents, unlike the membrane stiffening observed with longer electrosynthesis times. Figure $5 \mathrm{~b}, \mathrm{~d}$ shows that the initial electrolyte $\mathrm{pH}$ has little effect on relative monomeric and fibrillar composition of the resulting membranes.

Since the $\mathrm{pH}$ region that leads to less stiff membranes coincides with the isoelectric point for collagen in our experiments, ${ }^{15}$ this softening is likely related to pre-aggregation of collagen in the electrolyte, prior to incorporation in the membrane. As illustrated schematically in Figure 1, the non-specific aggregates could not mature into fibrils, and thus their incorporation would likely lead to a softer membrane. Earlier studies provide further support for these conclusions, showing that the specificity of collagen aggregates can be strongly affected by $\mathrm{pH}$ and concentration conditions. . $^{8,15,27,28}$

\section{Ion-dependent softening and stiffening}

Addition of biologically relevant ions to the electrolyte led to a range of Young's modulus changes, as shown in Figure 6. $\mathrm{Ca}^{2+}$ ions have been reported to enhance collagen aggregation during electrochemical processes ${ }^{29}$ and to accelerate both the nucleation and growth of collagen fibrils. ${ }^{30}$ Correspondingly, we observe larger Young's moduli (Figure 6a) after adding up to $10 \mathrm{mM} \mathrm{CaCl}_{2}$ to the starting electrolyte. On the other hand, $\mathrm{KCl}$ addition leads to substantially lower Young's 
moduli (Figure $6 \mathrm{~b}$ ), even though other reports have also suggested that $\mathrm{K}^{+}$promotes collagen aggregation. ${ }^{31}$ The changes we observe with the addition of $\mathrm{CaCl}_{2}$ and $\mathrm{KCl}$ contrast with the minimal effect that similar concentrations of $\mathrm{NaCl}$ have on stiffness (Figure 6c).
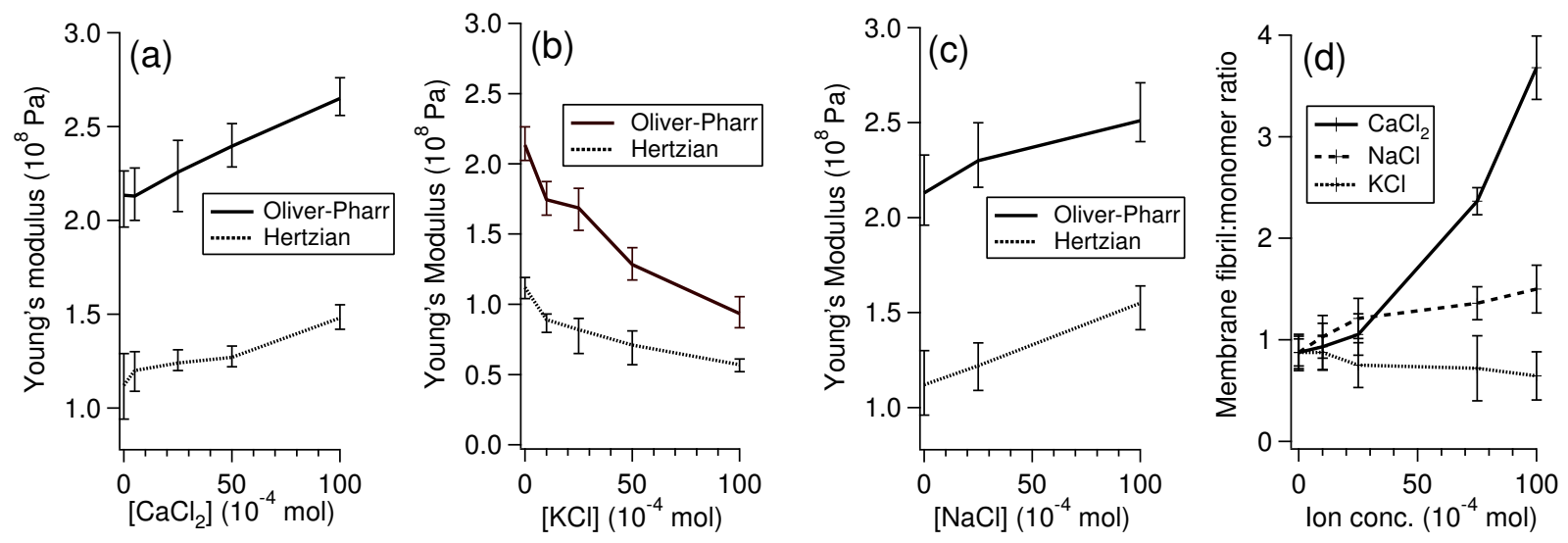

Figure 6: Young's moduli values can change dramatically with the addition of different concentrations of (a) $\mathrm{CaCl}_{2}$ or (b) $\mathrm{KCl}$, with smaller changes for the addition of (c) $\mathrm{NaCl}$ to the electrolyte. Stiffness trends follow changes in the relative amount of fibrillar collagen $(\mathrm{d}$, as determined from Raman scattering peak intensities), with stiffer membranes displaying higher fibril content.

These disparate stiffness trends as a function of ion concentration track very well with changes in the relative amounts of fibrillar and monomeric collagen (Figure 6d) as assessed from Raman scattering spectra. The stiffest membranes, prepared in electrolytes containing $10 \mathrm{mM} \mathrm{CaCl} 2$, display the highest relative fibril content. In contrast, membranes prepared in electrolytes with high $\mathrm{KCl}$ levels were mechanically weak and easily dissociable when rinsed in water, suggesting non-specific aggregation. Correspondingly, Raman peak intensities suggest a slightly lower fibril content.NaCl-containing electrolytes yield membranes with only slightly enhanced fibril content with increasing $\left[\mathrm{Na}^{+}\right]$(Figure 6c).

\section{Discussion and Conclusions}

With a correlation established between the degree of fibril content and the stiffness of the electrochemically synthesized collagen membranes, we investigated the points in the synthesis process at which fibril formation could occur, and how the coexistence of fibrils and monomers contributes 
to lateral uniformity in membrane stiffness.

Because collagen fibrillogenesis is a hierarchical process, ${ }^{5}$ the direct addition of monomers (from the electrolyte) cannot itself create more fibrils in the membrane (Figure 1). Instead, protofibrils must form at some point during the electrosynthesis process, either in the electrolyte or in the membrane itself. We see evidence of protofibrils in AFM images (provided in Supporting Information) of air-dried aliquots of electrolyte removed immediately prior to membrane formation, but qualitative comparisons based show that $\mathrm{Ca}^{2+}$-containing electrolyte (which produced the stiffest films with the highest relative fibril content) reveals very little fibrillar collagen relative to the $\mathrm{K}^{+}$containing electrolyte (which produced the softest films with the lowest relative fibril content). Since limited fibril formation in the electrolyte appears to correlate with higher fibril content in the membrane, and vice versa, it appears that fibrils must be able to grow within the membrane, and not just in the electrolyte. Additional support for this hypothesis, based on AFM experiments, is included in the Supporting Information.

Fibrils mature over time, so it is not surprising that longer electrosynthesis times lead to stiffer films. Similarly, it is reasonable to expect that initial electrolyte $\mathrm{pH}$ values close to the monomeric isoelectric point are more likely to promote non-specific aggregation at the expense of fibril formation, thereby leading to softer membranes. It is more complicated to explain the stiffness differences that arise from membranes prepared in the presence of different cations. $\mathrm{K}^{+}$and $\mathrm{Ca}^{2+}$ ions were selected because they have both been shown to promote collagen fibrillogenesis ${ }^{29-31}$ However, the cation effects on membrane stiffness are dramatically different, with increased $\mathrm{K}^{+}$ concentrations leading to collagen aggregation in solution, at the expense of fibril growth within the membrane. In contrast, $\mathrm{Ca}^{2+}$ promotes fibril maturation within the membrane, leading to higher fibril (rather than protofibril or monomer) content within the membrane and hence a higher Young's modulus. The precise role of these cations in the hierarchical assembly of collagen fibrils is not understood, although it has been suggested that the ions promote lateral aggregation of monomers or protofibrils through a range of mechanisms, including bridging or affecting the isoelectric point of the protein through binding to amino acid side chains. ${ }^{29-31}$ In the particular case of our reaction 
conditions, it appears that $\mathrm{Ca}^{2+}$ is required for higher-order fibril growth, perhaps due to its higher charge density (divalent and smallest of the three cations selected) allowing for better protofibril bridging.

Cation and $\mathrm{pH}$ differences aside, a remarkable feature of all electrochemically prepared membranes is that, despite the diversity of their constituent collagen aggregates (a mixture of fibrils and monomers), the spatial variation of the stiffness across the membrane is surprisingly consistent, with variations $\sim 10 \%$ (as seen in error bars on stiffness plots). This stiffness uniformity, in conjunction with AFM topographic images (Figure 7), suggest that the mechanical properties of the membranes rely on the presence of both fibrillar and monomeric collagen. Because we have established that monomeric collagen is continually added to the membrane during electrosynthesis, the top surface of the membrane that we image and probe with force microscopy has a predominant monomomeric component. Therefore, we rarely observe fibrillar signatures in topography images, even in the stiffest membranes. Underlying this monomer coating is a network of more mature collagen fibrils and protofibrils that provide greater stiffness. This idea is supported by AFM topography images on ethanol-rinsed samples for which the monomeric overlayer is partially removed, showing evidence of a dense and aligned network of fibrils in the stiffest membranes. However, it is interesting to note that the removal of the monomeric layer does not change the measured Young's modulus of the membrane, indicating that the monomer overlayer does not contribute significantly to the mechanical response of the membrane.

This work demonstrates that mechanical stiffness is a useful metric for characterizing complex collagen assemblies, providing insight about the relative importance of non-specific versus hierarchical aggregation products and pathways in collagen-based materials. The trends in the stiffness data are consistent whether the tip-membrane interactions are modeled with a Hertzian approach or the Oliver-Pharr model. Thus, we can be confident that the details of the tip-membrane interactions do not affect the conclusions we draw from the data. 


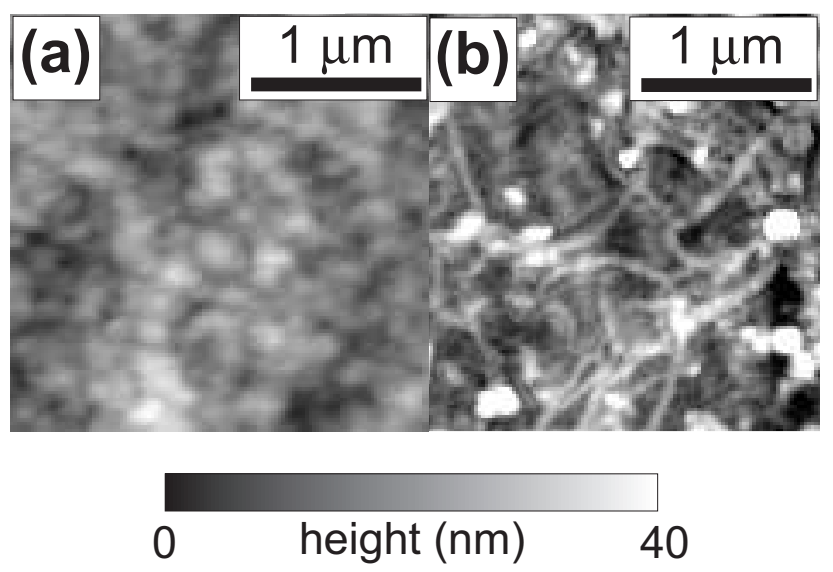

Figure 7: (a) A representative AFM height image of an as-synthesized collagen membrane shows rounded features with no obvious fibril signatures. (b) Rinsing with ethanol reveals a dense network of aligned fibrils within the membrane.

\section{Acknowledgment}

Thanks to Memorial's Department of Biochemistry for the use of their Raman spectroscopy facilities. We thank NSERC (Canada) and the Canada Foundation for Innovation for funding support.

\section{Supporting Information Available}

Supporting Information Available: Sample force curves and details regarding their analysis to extract Young's moduli, as well as atomic force microscopy images comparing fibril content in electrolyte and membrane for different durations of applied electric field. This material is available free of charge via the Internet at http://pubs.acs.org.

\section{References}

(1) Silver, F. H.; Christiansen, D. L.; Snowhill, P. B.; Chen, Y. Connect. Tissue Res. 2000, 41, 155-1-64.

(2) Silver, F. H.; Freeman, J. W.; Seehra, G. P. J. Biomech. 2003, 36, 1529-1553. 
(3) Cheng, X.; Gurkan, U. A.; Dehen, C. J.; Tate, M. P.; Hillhouse, H. W.; Simpson, G. J.; Akkus, O. Biomater. 2008, 29, 3278-3288.

(4) Guo, S. L.; Akhremitchev, B. B. Biomacromolecules 2006, 7, 1630.

(5) Kadler, K. E.; Holmes, D. F.; Trotter, J. A.; Chapman, J. A. Biochem. J. 1996, 316, 1-11.

(6) MacNeil, S. Nature 2007, 445, 874-880.

(7) Zani, B. G.; Kojima, K.; Vacanti, C. A.; Edelman, E. R. Proc. Nat. Acad. Sci. 2008, 105, 7046-7051.

(8) Köster, S.; Leach, J. B.; Struth, B.; Pfohl, T.; Wong, J. Y. Langmuir 2007, 23, 357-359.

(9) McDaniel, D.P.; Shaw, G. A.; Elliott, J. T.; Bhadriraju, K.; Meuse, C.; Chung, K.-H.; Plant, A. L.Biophys. J. 2007, 92, 1759-11769.

(10) Guo, C.; Kaufman, L. J. Biomater. 2007, 28, 1105-1114.

(11) Evans, H. J.; Sweet, J. K.; Price, R. L.; Yost, M.; Goodwin, R. L. Am. J. Physiol. Heart Circ. Physiol. 2003, 285, H570-H578.

(12) Lee, P.; Lin, R.; Moon, J.; Lee, L. P. Biomed. Microdevices 2006, 8, 35-41.

(13) Denis, F. A.; Pallandre, A.; Nysten, B.; Jonas, A. M.; Dupont-Gillain, C. C. Small 2005, 1, 984-991.

(14) Marino, A. A.; Becker, R. O. Calcified Tissue Res. 1970, 4, 330-338.

(15) Baker, H. R.; Merschrod S., E. F.; Poduska, K. M. Langmuir 2008, 24, 2970-2972.

(16) Matthews, J. A.; Wnek, G. E.; Simpson, D. G.; Bowlin, G. L. Biomacromol. 2002, 3, $232-238$.

(17) Hutter, J. L.; Bechhoefer, J. Rev. Sci. Instrum. 1993, 64, 1868-1873. 
(18) Akhremitchev, B. B.; Walker, G. C. Langmuir 1999, 15, 5630-5634.

(19) Hay, J. L.; Wolff, P. J. J. Mater. Res 2001, 16, 1280-1286.

(20) Pharr, G. M.; Oliver, W. C.; Brotzen, F. R. J. Mater. Res. 1992, 7, 613-617.

(21) Oliver, W. C.; Pharr, G. M. J. Mater. Res. 2004, 19, 3-20.

(22) Pharr, G. M.; Bolshakov, A. J. Mater. Res. 2002, 17, 2660-2671.

(23) Sasaki, N.; Odajima, S. J. Biomechanics 1996, 29, 655.

(24) van der Rijt, J. A. J.; van der Werf, K. O.; Bennink, M. L.; Dijkstra, J. P.; Feijen, J. J. Macromol. Biosci. 2006, 6, 697.

(25) Heim, A. J.; Matthews, G. W. Appl.Phys. Lett. 2006, 89, 181902.

(26) Wisniewski, M.; Sionkowska, A.; Kaczmarek, H.; Lazare, S.; Tokarev, V.; Belin, C. J. J. Photochem. Photobiol 2007, 182, 192-199.

(27) Huelin, S. D.; Baker, H. R.; Poduska, K. M.; Merschrod S., E. F. Macromolecules 2007, 40, 8440-8444.

(28) Gobeaux, F.; Mosser, G.; Anglo, A.; Panine, P.; Davidson, P.; Giraud-Guille, M.-M.; Belamie, E. J. Mol. Biol. 2008, 376, 1509-1522.

(29) Fan, Y.; Duan, K.; Wang, R. Biomater. 2005, 26, 1623-1632.

(30) Evans, C. H.; Drouven, B. J. Biochem. J. 1983, 213, 751-758.

(31) Freudenberg, U.; Behrens, S. H.; Welzel, P. B.; Müller, M.; Grimmer, M.; Salchert, K.; Taeger, T.; Schmidt, K.; Pompe, W.; Werner, C. Biophys. J. 2007, 92, 2108-2119. 


\section{for Table of Contents use only}

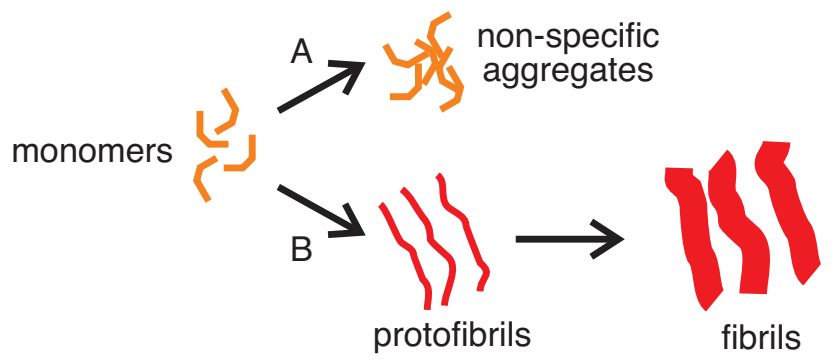

